\title{
Naturally occurring antibodies in cats against dog erythrocyte antigens and vice versa
}

\begin{tabular}{|c|c|}
\hline Journal: & Journal of Feline Medicine and Surgery \\
\hline Manuscript ID & JFMS-17-0059.R2 \\
\hline Manuscript Type: & Original Article \\
\hline Date Submitted by the Author: & 18-Jul-2017 \\
\hline Complete List of Authors: & $\begin{array}{l}\text { Priolo, Vito; Universita degli Studi di Messina, Department of Veterinary } \\
\text { Science } \\
\text { Masucci, Marisa; Universita degli Studi di Messina Dipartimento di Scienze } \\
\text { Veterinarie } \\
\text { Spada, Eva; Universita degli Studi di Milano, Department of Veterinary } \\
\text { Science } \\
\text { Proverbio, Daniela; Universita degli Studi di Milano, Department of } \\
\text { Veterinary Science } \\
\text { Pennisi, Maria Grazia ; Università di Messina, Scienze Veterinarie }\end{array}$ \\
\hline Keywords: & $\begin{array}{l}\text { feline, dog erythrocyte antigen (DEA), xenotransfusion, blood type, cross- } \\
\text { matching tests, cat erythrocyte antigen, canine }\end{array}$ \\
\hline Abstract: & $\begin{array}{l}\text { Objectives } \\
\text { The aim of this study was to investigate the presence of naturally occurring } \\
\text { antibodies against canine erythrocyte antigens in cats, and vice versa. The } \\
\text { influence of canine and feline blood type on cross-match results was also } \\
\text { studied. } \\
\text { Methods } \\
\text { Blood samples from } 34 \text { cats and } 42 \text { dogs were used to perform test-tube } \\
\text { major and minor cross-match tests and blood typing. Blood from each cat } \\
\text { was cross-matched with blood from two to six dogs, for a total of } 111 \\
\text { cross-match tests. Hemolysis, macro- and/or micro-agglutination were } \\
\text { considered markers of a positive cross-match. } \\
\text { Results } \\
\text { Eighty-three overall major cross-match tests were positive at } 37^{\circ} \mathrm{C}, 86 \text { at } \\
\text { room temperature and } 90 \text { at } 4^{\circ} \mathrm{C} \text {. The minor cross-match tests were } \\
\text { positive in all but two cross-matches performed at } 37^{\circ} \mathrm{C} \text {, all tests } \\
\text { performed at room temperature and all but one test performed at } 4^{\circ} \mathrm{C} \text {. No } \\
\text { cats tested totally negative at both major and minor cross-matches } \\
\text { performed with samples from any single dog. Prevalence of warm natural } \\
\text { antibodies against canine erythrocyte antigens was lower in type B cats } \\
\text { compared to A, regardless of the blood type of donor dogs. } \\
\text { Conclusions and relevance } \\
\text { This study reveals a high prevalence of naturally-occurring antibodies in } \\
\text { cats against dog erythrocyte antigens, and vice versa, and suggests that } \\
\text { transfusion of cats with canine blood is not recommended as a routine } \\
\text { procedure due to the potential high risk of either acute severe or milder } \\
\text { transfusion reactions. }\end{array}$ \\
\hline
\end{tabular}


SCHOLARONE $^{\text {m }}$
Manuscripts 
Replies to Reviewers' Comments to Author:

Reviewer: 2

Reviewers report for the author

Acceptable for publication with minor grammatical corrections only.

Minor corrections are written on scanned copy for use by authors and editor.

ANSWER: all corrections written on the scanned copy have been made.

We changed "cross-matching" to "cross-match" as suggested at lines 22 and 33 and also in the whole manuscript for consistency.

Other minor corrections:

Line 58: Say "(such as the Mik system)"

ANSWER: done

Line 282: Say "...does not predict an absence of reactions against leukocytes...."

ANSWER: done

Line 293: Say "....or that the prevalence of alloantibodies and feline blood types vary....."

ANSWER: done

Tables 1 and 2: The two tables are formatted differently. Table 1 would say, for example, 75, whereas Table 2 would say 75/101. Reformat one of the tables so that they both are formatted comparably.

ANSWER: we modified Table 1 as suggested

Reviewer: 1

Reviewers report for the author

The authors have addressed the comments by both reviewers very well and in an exceptionally clear way.

The research is much clearer and reads very well to me.

Just a few very minor edits on the attached pdf.

ANSWER: all edits indicated on the attached pdf have been accepted.

As required at line 31, we checked the number of major XM positive at room temperature (\# 86) and it is correct as well as at line 189 and in Table 1 
1 Naturally occurring antibodies in cats against dog erythrocyte antigens and vice versa

2

3 Vito Priolo ${ }^{1}$, Marisa Masucci ${ }^{1}$, Eva Spada ${ }^{2}$, Daniela Proverbio ${ }^{2}$, Maria Grazia

$4 \quad$ Pennisi ${ }^{1}$

5 'Department of Veterinary Science, University of Messina, Polo Universitario

6 Annunziata 98168, Messina, Italy

$7 \quad{ }^{2}$ Veterinary Transfusion Research Laboratory (REVLab), Department of

8 Veterinary Medicine, University of Milan, Via G. Celoria 10 - 20133 Milan, Italy

9

10 Corresponding author: Marisa Masucci DVM, PhD Department of Veterinary

11 Science, University of Messina, Polo Universitario Annunziata 98168, Messina,

12 Italy

13 E-mail: marisa.masucci@unime.it telephone: 00390903503740

14

15 Key words: blood type, cross-matching tests, dog erythrocyte antigen (DEA),

16 xenotransfusion, cat erythrocyte antigen, feline, canine 
18 Abstract

19 Objectives

20 The aim of this study was to investigate the presence of naturally occurring

21 antibodies against canine erythrocyte antigens in cats, and vice versa. The

22 influence of canine and feline blood type on cross-match results was also

23 studied.

24 Methods

25 Blood samples from 34 cats and 42 dogs were used to perform test-tube major

26 and minor cross-match tests and blood typing. Blood from each cat was cross-

27 matched with blood from two to six dogs, for a total of 111 cross-match tests.

28 Hemolysis, macro- and/or micro-agglutination were considered markers of a

29 positive cross-match.

30 Results

31 Eighty-three overall major cross-match tests were positive at $37^{\circ} \mathrm{C}, 86$ at room

32 temperature and 90 at $4^{\circ} \mathrm{C}$. The minor cross-match tests were positive in all but

33 two cross-matches performed at $37^{\circ} \mathrm{C}$, all tests performed at room temperature 
34 and all but one test performed at $4^{\circ} \mathrm{C}$. No cats tested totally negative at both

35 major and minor cross-matches performed with samples from any single dog.

36 Prevalence of warm natural antibodies against canine erythrocyte antigens was

37 lower in type B cats compared to A, regardless of the blood type of donor dogs.

38 Conclusions and relevance

39 This study reveals a high prevalence of naturally-occurring antibodies in cats

40 against dog erythrocyte antigens, and vice versa, and suggests that transfusion

41 of cats with canine blood is not recommended as a routine procedure due to the

42 potential high risk of either acute severe or milder transfusion reactions. 


\section{Introduction}

44 Two feline blood group systems are known: AB (comprising types A, B and $45 \mathrm{AB}$ ), and Mik (including types Mik positive and Mik negative). ${ }^{1}$ Type A cats 46 may have weak natural anti-B alloantibodies. In contrast type B cats have 47 strong natural anti-A alloantibodies, causing acute, severe hemolytic reactions 48 against type A erythrocytes. Type $\mathrm{AB}$ cats do not have natural alloantibodies. ${ }^{2}$

49 The Mik blood group system was recently identified in USA. ${ }^{3}$ Mik negative cats 50 can have naturally occurring anti-Mik alloantibodies that elicit acute hemolytic 51 transfusion reactions. ${ }^{3}$ Therefore accurate identification of blood types is 52 important in feline practice to reduce the possibility of potentially fatal 53 transfusion reactions and obtain the best efficacy from blood transfusions. ${ }^{4}$

54 While several feline $\mathrm{AB}$ typing kits are commercially available for clinical 55 practice, typing of $\mathrm{AB}$ and $\mathrm{B}$ cats can still pose challenges because erroneous 56 and discordant blood typing results have been reported in cats. ${ }^{4,5}$ Furthermore, 57 they cannot account for antigens outside of the AB system (such as the Mik 58 system) nor for alloantibodies present in the recipient. ${ }^{6}$ The prevalence of non59 AB blood types is unknown at present. Two recent studies, based on a limited 
60 number of cats, did not find evidence for non-AB blood type incompatibilities. ${ }^{4,6}$

61 When possible, cross-match $(\mathrm{XM})$ that detects recipient antibodies against

62 donor erythrocytes (major $\mathrm{XM}$ ) and donor antibodies against recipient

63 erythrocytes (minor XM) should be performed prior to transfusion to increase

64 patient safety. 2,6

65 Blood transfusion in the feline species may be challenging. In fact, the small size

66 of donors makes blood collection technically more difficult than in dogs, and

67 sedation is usually required for bleeding donors. Moreover, the high prevalence

68 of naturally-occurring alloantibodies against feline red blood cell (RBC)

69 antigens demands that blood typing is performed before any transfusion, and

70 the need to use donors and recipients of the same blood type can make

71 transfusions difficult in cats with rare blood types, such as B or AB. ${ }^{1,2,7}$

72 Despite xenotransfusions being abandoned in all other domestic species since

73 the early 1900s, transfusion of canine blood to cats is still performed in

74 veterinary practice as a life-saving procedure when hemoglobin-based oxygen

75 carrier solutions are not available and a suitable feline donor cannot be

76 found. $5,8,9,10$ 
77 Based on a limited number of cases reported in the veterinary literature, with 78 most publications dating from 1960s, cats did not appear to have naturally79 occurring antibodies against canine RBC antigens. ${ }^{8}$ However, a recent study 80 reported significant incompatibilities detected by $\mathrm{XM}$ between feline and canine 81 blood. ${ }^{5}$ No severe acute adverse reactions have been described for cats 82 receiving a single transfusion with canine blood. $5,8,9,11,12$ Only mild transfusion 83 reactions occasionally occurred during the transfusion or in the following 84 week. ${ }^{5,8}$ In most reports, cats transfused with canine blood improved 85 clinically. 5,9,10,13 However, antibodies against canine RBCs were produced within 86 4-21 days of the transfusion, and any repeated transfusion with canine blood 87 later than 6 days after the first one caused severe acute reactions which were 88 frequently fatal. 811,12 Moreover, the lifespan of the transfused canine RBCs was 89 very short (3-5 days). ${ }^{5,14}$

90 Because of the limited number of cases reported in the literature, more data are 91 needed to evaluate the benefit and the risks of dog-to-cat xenotransfusions.

92 The purpose of this study was to assess the potential risk of adverse transfusion 93 reactions in cats transfused with canine blood, by evaluating the occurrence of 
94 feline naturally occurring antibodies against canine RBC antigens and vice versa.

95 The influence of blood types of cats and dogs on XM results was also

96 investigated.

97 Materials and methods

98 Samples

99 Surplus material from diagnostic samples of 34 domestic shorthair cats and 42

100 dogs of 17 different breeds admitted to the Teaching Veterinary Hospital of

101 University of Messina for elective surgery, annual health check or health

102 problems between February and November 2015 was used. Informed consent

103 was obtained from owners and results from blood typing were offered to them

104 free of charge. About $1 \mathrm{~mL}$ of $\mathrm{K}_{2}$ EDTA-blood and, when available, up to $1 \mathrm{~mL}$

105 of blood serum were used to perform blood typing and XM tests. Hemolyzed

106 samples were excluded from the study. Blood was stored at $4^{\circ} \mathrm{C}$ until use and

107 was brought to room temperature (RT) before testing. Cross-match and canine

108 blood typing were performed within 24 hours after blood collection. Feline

109 blood typing was performed within a week after blood collection. 


\section{Blood typing}

111 The canine DEA 1 system was typed using a commercial immuno-

112 chromatographic test (Lab test DEA1- Alvedia, Limonest, France) according to 113 the manufacturer procedure.

114 Blood typing of all cats was determined at Veterinary Transfusion Research 115 Laboratory (REVLab) Unit, Department of Veterinary Medicine, University of 116 Milan, Italy using a tube agglutination method and confirmed with a back117 typing technique. ${ }^{15}$ EDTA-blood (150 $\left.\mu \mathrm{L}\right)$ was centrifuged for 2 mins at 1,000 $\mathrm{x}$ $118 \mathrm{~g}$ at RT. Plasma was removed and the RBC pellet was resuspended in $5 \mathrm{~mL}$ of 119 saline solution $(0.9 \% \mathrm{NaCl})$ and washed three times by repeating centrifugation, 120 discharge of supernatant and addition of PBS. Finally, $25 \mu \mathrm{L}$ of a 5\% RBCs PBS 121 suspension were put in three tubes and mixed respectively with: $50 \mu \mathrm{L}$ of type 122 B serum (anti-A reagent), $8 \mu \mathrm{g}$ of Triticum vulgaris lectin/mL in PBS solution 123 (anti-B reagent), or saline solution $(0.9 \% \mathrm{NaCl})$. These mixtures were incubated 124 at RT for 15 mins before centrifugation for $15 \mathrm{~s}$ at $1,000 \times \mathrm{g}$. Tubes were then 125 gently shaken, checked for agglutination and considered positive if 126 macroscopic agglutinates were observed. The cats were considered type A if 
127 agglutination was detected in the tube containing anti-A reagent, type B when 128 agglutination was observed in the tube containing anti-B reagent, and type $A B$ 129 if agglutination was seen in both tubes. Alloantibody testing was performed in 130 all type $\mathrm{B}$ or $\mathrm{AB}$ samples to detect the presence or absence of alloantibodies. 131 When a sample appeared to be $A B$ or $B$, it was confirmed with the back typing 132 technique: washed 5\% RBC suspension from the test sample, a known type A 133 cat and a known type B cat were incubated with the plasma sample as 134 described for tube agglutination to detect the presence (in type B cats versus 135 type $\mathrm{A}$ RBCs) or absence (in type $\mathrm{AB}$ cats either versus type $\mathrm{A}$ and type $\mathrm{B} \mathrm{RBCs}$ ) 136 of alloantibodies.

\section{Cross-match tests}

138 Cross-match procedures were always performed by the same experienced 139 technicians, and checked by one of authors (MM). ${ }^{16,17}$

$140 \mathrm{~K}_{2}$ EDTA tubes were centrifuged to separate RBCs from plasma, which were 141 transferred to separate tubes. Cat (recipient) and dog (donor) RBCs were 142 washed three times by adding about $1 \mathrm{ml}$ of saline solution $(0.9 \% \mathrm{NaCl})$, mixing 143 gently and centrifuging at 1,000 x $\mathrm{g}$ for $1 \mathrm{~min}$, then removing supernatant. Five 
144 percent donor and recipient RBC suspensions in saline solution were then 145 prepared. When the amount of left over samples was scant, priority was given 146 to perform major $\mathrm{XM}$ testing, and to perform incubations at $37^{\circ} \mathrm{C}$ because both 147 these evaluations are considered more relevant for predicting severe post148 transfusion reactions in the recipient animal. ${ }^{17}$ EDTA plasma was used when 149 serum was insufficient or hemolytic.

151 An equal amount of donor RBC suspension and recipient serum or plasma were 152 placed in three tubes, mixed and incubated respectively at $4^{\circ} \mathrm{C}$ and RT for 30 153 mins, and at $37^{\circ} \mathrm{C}$ for 15 mins. ${ }^{16}$ The tubes were then centrifuged at $115 \mathrm{x}$ for 1 $154 \mathrm{~min}$ and the supernatant was evaluated for hemolysis. Tubes were then shaken 155 gently to re-suspend cells and check for macroagglutination. If no obvious 156 agglutination was observed in the tube, one drop of blood suspension was 157 placed on a glass slide and examined for evidence of microagglutination. 158 Hemolysis, macro and/or microagglutination were considered markers of a 159 positive XM. 
161 Minor XM, donor and recipient controls were respectively performed as 162 described for major XM by mixing recipient RBC suspension and donor serum 163 or plasma (minor XM), donor RBC suspension and donor serum or plasma 164 (donor control), or recipient RBC suspension and recipient serum or plasma 165 (recipient control). The controls were performed for all samples, apart from one 166 cat, and only at RT.

\section{Statistical analyses}

168 Statistical analyses were performed using the GraphPad InStat v3.05 statistic 169 program (GraphPad Software Inc., San Diego California, USA, 2000) for 170 Windows 95. The Fisher's exact test was used to determine whether there were 171 statistical differences: a) in frequency of hemolysis or agglutination according to 172 temperature of incubation, both in the major $\mathrm{XM}$ and minor XM tests; $\mathrm{b}$ ) in 173 frequency of positive results (hemolysis and/or agglutination) according to the 174 recipient and donor blood type in the major XM test at the three temperatures 175 of incubation. P values $\leq 0.05$ were considered significant.

176 Results 


\section{Blood typing}

178 Fifteen dogs were DEA1 negative, 12 were DEA1 strong positive and 15 were

179 DEA1 weak positive. ${ }^{18}$

180 Twenty-seven cats were type A, three type B and four type AB. All type B and

181 AB samples were confirmed by back typing.

182 Cross-match tests

183 Blood from each cat was cross-matched with blood from a variable number of

184 dogs ranging from two to six, for a total of 111 cross-matches. Ninety-seven 185 complete $\mathrm{XM}$ tests including major $\mathrm{XM}$ and minor $\mathrm{XM}$ at the three different 186 temperatures of incubation were obtained. Major XM was not performed in 187 seven cases at both $4^{\circ} \mathrm{C}$ and $\mathrm{RT}$, and minor XM was not done in 10 cases at $4^{\circ} \mathrm{C}$ 188 and RT and in four cases at $37^{\circ} \mathrm{C}$. Eighty-three/111 (74.8\%) overall major XM 189 tests proved positive at $37^{\circ} \mathrm{C}, 86 / 104(82.6 \%)$ at $\mathrm{RT}$ and $90 / 104(86.5 \%)$ at $4^{\circ} \mathrm{C}$. 190 Details about detection of hemolysis and/or agglutination are given in Table 1. 191 The minor $\mathrm{XM}$ tests were positive in all but two $\mathrm{XMs}$ performed at $37^{\circ} \mathrm{C}$ $192(98.1 \%)$, all tests performed at RT (100\%) and all but one test performed at $4^{\circ} \mathrm{C}$ 
193 (99\%). Details about detection of hemolysis and/or agglutination are given in 194 Table 2. No cats tested totally negative for both major XM and minor XM 195 procedures performed using samples from any single matched dog. Major XM 196 was negative at all three temperatures only in 2/104 (1.9\%) tests, was negative at 197 both $37^{\circ} \mathrm{C}$ and RT in $9 / 104(8.6 \%)$ tests, and was negative at $37^{\circ} \mathrm{C}$ only in $28 / 111$ $198(25.2 \%)$ tests. In major XM tests, hemolysis was significantly more frequent at $19937^{\circ} \mathrm{C} \quad(21 / 111=18.9 \%)$ compared to $\mathrm{RT}(9 / 104=8.6 \%) \quad(\mathrm{P}=0.032)$ and $4^{\circ} \mathrm{C}$ $200(5 / 104=4.8 \%)(P=0.0015)$. Conversely, agglutination was significantly more 201 frequent at $4^{\circ} \mathrm{C}(88 / 104=84.6 \%)$ compared to $37^{\circ} \mathrm{C}(71 / 111=63.9 \%)(\mathrm{P}=0.0006)$, 202 and at $\operatorname{RT}(81 / 104=77.9 \%)$ compared to $37^{\circ} \mathrm{C}(\mathrm{P}=0.0354)$. For minor $\mathrm{XM}$ tests, 203 there was no significant difference in frequency of hemolysis or agglutination 204 according to temperatures of incubation.

205 Cross-match of each single cat showed different patterns of compatibility 206 towards the two to six tested canine samples.

207 Cross-match results based on feline and canine blood type typing

208 Results of major XM based on canine and feline blood types are reported in 209 Table 3. Significant differences were found only at $37^{\circ} \mathrm{C}$ for the two following 
210 combinations: a) feline type A with canine DEA1 strong positive (positive 211 reactions: $31 / 36=86.1 \%$ ) in comparison to feline type B with canine DEA1 strong 212 positive (positive reactions: $2 / 6=33.3 \%)(\mathrm{P}=0.01)$; b) feline type A with canine 213 DEA1 strong positive (positive reactions: $31 / 36=86.1 \%$ ) in comparison to feline 214 type B with canine DEA1 negative (positive reactions: 1/4=25\%) $(\mathrm{P}=0.02)$.

215 Discussion

216 This study reveals a high prevalence of naturally occurring antibodies in cats 217 against dog erythrocyte antigens, and vice versa. In fact, no tested cat was totally 218 negative for hemolysis and/or agglutination for both major and minor XM 219 procedures performed at $4^{\circ} \mathrm{C}$, RT and $37^{\circ} \mathrm{C}$ with samples from any single $\mathrm{dog}$.

220 The presence of hemolysis or agglutination on major and minor XM testing 221 implies that the recipient is not compatible, respectively, to the donor's RBCs or 222 to the donor's plasma. ${ }^{19}$ The presence of macroagglutination and hemolysis on 223 major XM precludes the use of the donor's RBCs because it indicates that, in the 224 recipient, a severe adverse acute transfusion reaction may occur. ${ }^{11,20}$ Conversely, 225 the presence of microagglutination may not necessarily indicate that the patient 226 will have a severe adverse transfusion reaction. ${ }^{15}$ It is commonly accepted that 
227 blood for transfusion ideally should be compatible at $37^{\circ} \mathrm{C}$ and $\mathrm{RT}$, but major $228 \mathrm{XM}$ at $37^{\circ} \mathrm{C}$ is clinically the most important compatibility. ${ }^{17}$ However, cold $\left(4^{\circ} \mathrm{C}\right)$ 229 incompatibilities can cause microthrombosis in acral capillary beds and 230 therefore potentially ischemic necrosis of the tip of ears, nose or tail during cold 231 weather. ${ }^{21}$

232 In 57.6\% (64/111) of major $\mathrm{XM}$ tests that we performed at $37^{\circ} \mathrm{C}$, hemolysis 233 and/or macroagglutination were found, suggestive of a high risk of severe acute 234 transfusion reactions. ${ }^{17}$ Moreover, feline hemolysins against dog RBCs were 235 more prevalent at $37^{\circ} \mathrm{C}$, conversely hemoagglutinins were more prevalent at $2364^{\circ} \mathrm{C}$. A limitation of this study is the lack of controls at $4^{\circ} \mathrm{C}$ and at $37^{\circ} \mathrm{C}$, due to 237 the restricted amount of available blood. Because of this, positive results at 238 these incubation temperatures could have been overestimated. Furthermore, 239 hemolytic reactions could have been underestimated when XMs were 240 performed using plasma obtained from EDTA blood. In fact, complement 241 activation is responsible for in vitro hemolysis after anti-RBC antibodies reacted 242 with RBC antigens, but it cannot occur when calcium and magnesium cations 243 are chelated by EDTA. ${ }^{22}$ 
244 Further limitations of this study are that we did not test cats for the Mik system 245 group, and we had the opportunity to test very few feline type $B$ and $A B$ 246 samples because of their low prevalence in the feline population. ${ }^{23,24}$ However, 247 the prevalence of warm natural antibodies against canine RBCs was lower in 248 type B cats compared to type A only when matched with DEA1 strong positive 249 blood. We can therefore assume that type A cats more frequently have warm 250 natural antibodies against DEA1 strong positive RBCs and could have a higher 251 risk for severe acute adverse reactions after xenotransfusion with DEA1 strong 252 positive donors.

253 Almost all minor XM tests in this study were positive, and mostly agglutination 254 reactions were detected. When the volume of donor plasma transfused is small, 255 antibodies in donor plasma become significantly diluted in the recipient blood 256 stream, and therefore the results of the minor XM test may not be clinically 257 relevant or may cause mild to moderate acute transfusion reactions. ${ }^{17}$ However 258 transfusion of large amounts of canine whole blood containing antibodies 259 against the recipient's RBCs may cause severe hemolysis and worsen a pre260 existing anemia. ${ }^{8}$ This could occur as a result of repeated whole blood 
261 transfusions in subsequent days or of administration of large amounts of 262 plasma.

263 Extensive data about pre-transfusion dog-to-cat XM tests are not available. In 264 fact, published studies report information regarding XM tests in about 56 cases 265 only.5,8 Nineteen cats showed agglutination against canine red blood cells on 266 major $\mathrm{XM}$, and in only two cases on minor XM. ${ }^{8}$ Unfortunately, all these tests 267 were performed at one temperature of incubation only: RT or $37^{\circ} \mathrm{C}$. Moreover, 268 minor XM, microagglutination or hemolysis were usually not evaluated.5,8,11 269 Microagglutination and incompatibility reactions in major XM at RT or in 270 minor XM can cause milder reactions and reduce the survival of transfused red 271 blood cells. This could be the reason why mild transfusion reactions have 272 previously been reported occasionally during the transfusion or in the 273 following week. ${ }^{8,20}$ Furthermore, in some studies the lifespan of transfused 274 canine RBCs was shortened to less than 4-5 days compared to a 30 days half-life 275 for compatible feline RBCs $5,14,25$

276 Negative major and/or minor $\mathrm{XM}$ tests do not completely eliminate the risk 277 associated with transfusions, and do not guarantee an expected lifespan of 
278 transfused erythrocytes, because delayed reactions can be caused by the 279 production of antibodies against RBC antigens shortly after the transfusion. ${ }^{26}$ 280 Additionally, a negative RBC XM test does not predict an absence of reactions 281 against leukocytes and plasma proteins. ${ }^{26}$ Therefore, although $\mathrm{XM}$ tests are 282 considered to be the standard test for assessing the risk of blood transfusion 283 due to immunological reactions in practice, they are not fully predictive of the 284 risk of transfusion reactions. ${ }^{5}$

285 This study, as also recently found by Euler et al (2016), consistently shows a 286 high degree of incompatibility when dog and cat blood are cross-matched. 287 Despite this, reports of acute transfusion reactions on first transfusion of dog 288 blood to cats are rare, according to both publications dating from the 1960s and 289 a few recent case reports.5,9,10,11,12,13,25 The discrepancy between multiple reported 290 safe dog-to-cat transfusions and consistent XM incompatibility could be due to 291 the fact that natural alloantibodies have changed over time, or that the prevalence of alloantibodies and feline blood types vary in different geographic 293 areas, or that the older studies missed minor transfusion reactions. Finally, a 294 low positive predictive value for adverse xenotransfusion reactions following 
295 incompatible dog-cat XM cannot be excluded, but this positive predictive value 296 cannot be explored in clinical settings, because blood is almost never transfused 297 when a positive XM is obtained and, in emergency situations, cats are 298 presumably transfused without performing XM with the donor dog.

\section{Conclusions}

300 Transfusion of cats with canine blood is not recommended as a routine 301 procedure because the high prevalence of XM incompatibilities theoretically 302 suggests an elevated risk of severe acute reactions or of milder reactions that 303 make the xenotransfusion less beneficial than transfusion with matched feline 304 whole blood. In exceptional circumstances where xenotransfusion is the only 305 means available for the short-term stabilization of a feline patient until 306 obtaining compatible feline blood or bone marrow red cell regeneration, XM 307 tests should always be performed. A completely compatible canine blood 308 might be extremely difficult to find and, in this case, dogs found negative at 309 major $\mathrm{XM}$ (best at $37^{\circ} \mathrm{C}$ ) would be preferred. 
311 The authors thanks Angela Burrascano and Elisa Zanghì for laboratory

312 technical support in performing XM tests.

313 Supplementary material

314 Table 1, table 2, table 3

315 Author note:

316 This paper was presented in part at the 25 $5^{\text {th }}$ ECVIM-CA Congress 2015 in

317 Lisbon (Portugal)

318 Conflict of interest

319 The authors declare no potential conflicts of interest with respect to the 320 research, authorship, and/or publication of this article.

$321 \quad$ Funding

322 The authors received no financial support for the research, authorship, and/or 323 publication of this article.

324 References 
1. Barfield D and Adamantos S. Feline blood transfusions: A pinker shade of pale. J Feline Med Surg 2011; 13: 11-23.

2. Kisielewicz $C$ and Self IA. Canine and feline blood transfusions: 
Feline Med Surg Epub ahead of print 19 January 2017. DOI: $10.1177 / 1098612 \times 16688574$.

7. Bracker KE and Drellich S. Transfusion reactions. Compend Contin Educ Vet 2005; 27: 500-517.

8. Bovens C and Gruffydd-Jones T. Xenotransfusion with canine blood in the feline species: Review of the literature. J Feline Med Surg 2012; 15: 62-67. DOI: $10.1177 / 1098612 \times 12460530$

9. Oron L, Bruchim Y, Klainbart S, et al. Ultrasound-guided intracardiac xenotransfusion of canine packed red blood cells and epinephrine to the left ventricle of a severely anemic cat during cardiopulmonary resuscitation. J Vet Emerg Crit Care 2017; 27: 218-223.

10. Sárpataki O, Bedeceani $\mathrm{j}$, Codea $\mathrm{R}$, et al. Blood transfusion with canine blood in two cats with hypovolemic anemia. Bulletin UASVM Vet Med 2014; 71: 513-514. DOI:10.15835/buasvmcn-vm: 10622

11. Hessler J, Davis LE and Dale HE. Effect of repeated transfusions of dog blood to cats. Small An Clin 1962; 2: 684-687. 
12. Lautié R, Coulon j, Geral MF, et al. Blood hetero-transfusion in the cat. Immunological and clinical study. Rev Med Vet 1969; 120: 311-323 [in French]

13. Weingram T. Xenotransfusion of canine blood to a cat. Isr J Vet Med 2014; 69: 50-52.

14. Clark $\mathrm{CH}$ and Kiesel GK. Longevity of red blood cells in interspecies transfusion. J Am Vet Med Assoc 1963; 143: 400-401.

15. Seth M, Jackson KV, Giger U, et al. Comparison of five blood-typing methods for the feline AB blood group system. Am J Vet Res 2011; 72: 203-209. DOI: 10.2460/ajvr.72.2.203.

16. Bull RW. Immunohematology. In: Halliwell REW and Gorman NT (eds) Veterinary clinical immunology. Philadelphia: WB Saunders Company, 1989, pp. $467-472$.

17. Abrams-Ogg ACG. Practical blood transfusion. In: Day MJ, Mackin AJ and Littlewood J (eds). BSAVA Manual of canine and feline haematology and transfusion medicine. Gloucester: British Small Animal Veterinary Association, 2000, pp 263-303. 
18. Acierno MM, Raj K and Giger U. DEA 1 Expression on dog erythrocytes analyzed by immunochromatographic and flow cytometric techniques. J Vet Intern Med 2014; 28: 592-598. DOI: 10.1111/jvim.12321.

19. Prittie JE. Triggers for use, optimal dosing, and problems associated with red cell transfusions. Vet Clin North Am Small Anim Pract 2003; 33: $1261-1275$.

20. Giger U and Bücheler J. Transfusion of type-A and type-B blood to cats. J Am Vet Med Assoc 1991; 198: 411-418.

21. Day M. Immune mediated haematological disease. In: Day $M$ and Mackin AJ (eds). Clinical immunology of the dog and cat. 2th ed. Boca Raton: Manson Publishing, 2012, pp 94-120.

22. Brechter ME. Red cell antigen-antibody reactions and their detection. In: Brechter ME AABB Technical Manual Program Unit. 15th ed. Bethesda, Maryland, USA: American Association of Blood Banks, 2005, pp. 271-287.

23. Fosset FTJ and Blais MC. Prevalence of feline blood groups in the Montreal area of Quebec, Canada. Can Vet J 2014; 55: 1225-1228. 
392

24. Proverbio D, Spada E, Perego R, et al. Comparison of gel column agglutination with monoclonal antibodies and card agglutination methods for assessing the feline $A B$ group system and a frequency study of feline blood types in northern Italy. Vet Clin Pathol 2011; 40: 3239.

25. Marion RS and Smith JE. Survival of erythrocytes after autologous and allogenic transfusion in cats. J Am Vet Med Assoc 1983; 183: 1437-1439.

26. Tocci LJ and Ewing $P$. Increasing patient safety in veterinary transfusion medicine: an overview of pretransfusion testing. $J$ Vet Emerg Crit Care 2009; 19: 66-73. DOI: 10.1111/j.1476-4431.2009.00387.x. 
1 Tables

2 Table 1: Results (agglutination and/or hemolysis) of major XM test at the three temperatures of incubation.

3 The number of agglutinations detected microscopically only is indicated in brackets

\begin{tabular}{lccc} 
Type of result & $4^{\circ} \mathrm{C}$ & $\mathrm{RT}$ & $37^{\circ} \mathrm{C}$ \\
\hline $\begin{array}{l}\text { Negative for hemolysis and } \\
\text { agglutination }\end{array}$ & $14 / 104$ & $18 / 104$ & $28 / 111$ \\
\hline $\begin{array}{l}\text { Hemolysis positive and } \\
\text { agglutination negative }\end{array}$ & $2 / 104$ & $5 / 104$ & $12 / 111$ \\
\hline $\begin{array}{l}\text { Hemolysis negative and } \\
\text { agglutination positive }\end{array}$ & $85 / 104(13)$ & $77 / 104(12)$ & $62 / 111(18)$ \\
\hline $\begin{array}{l}\text { Positive for hemolysis and } \\
\text { agglutination }\end{array}$ & $3 / 104(1)$ & $4 / 104(0)$ & $9 / 111(1)$ \\
\hline Total & $104(14)$ & $104(12)$ & $111(19)$
\end{tabular}

4

5 
6 Table 2: Results (agglutination and/or hemolysis) of minor XM at the three temperatures of incubation. The

7 number of agglutinations detected microscopically only is indicated in brackets. *This denominator is less 8 than the total number reported in the column because in some cases all RBCs were destroyed by hemolysis,

9 and it was not possible to evaluate agglutination

\begin{tabular}{llll} 
Type of result & $4^{\circ} \mathrm{C}$ & $\mathrm{RT}$ & $37^{\circ} \mathrm{C}$ \\
\hline $\begin{array}{l}\text { Negative for hemolysis and } \\
\text { agglutination }\end{array}$ & $1 / 101$ & $0 / 101$ & $2 / 107$
\end{tabular}

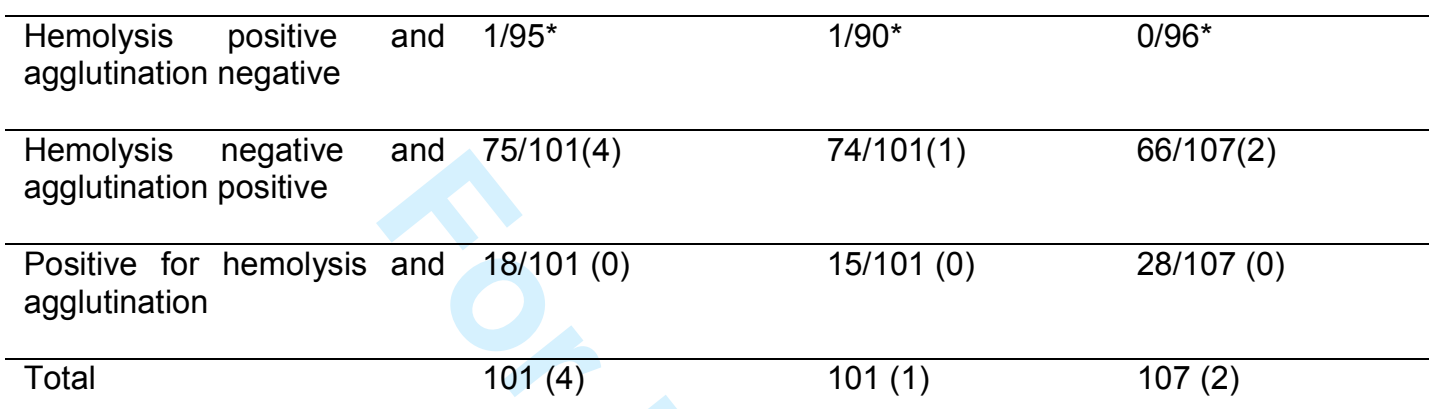

10

11 
12 Table 3: Results of major XM tests at the three temperatures of incubation according to feline blood type and

13 DEA classification of canine blood. $\mathrm{P}$ : positive hemolysis and/or agglutination; $\mathrm{N}$ : negative hemolysis and 14 agglutination, BT: blood type

\begin{tabular}{|c|c|c|c|c|c|c|c|}
\hline Cat BT & Dog BT & $4^{\circ} \mathrm{C}$ & & RT & & $37^{\circ}$ & \\
\hline & & $\mathrm{P}$ & $\mathrm{N}$ & $P$ & $\mathrm{~N}$ & $\mathrm{P}$ & $\mathrm{N}$ \\
\hline$A$ & $\begin{array}{l}\text { DEA } 1 \\
\text { Strong + }\end{array}$ & $29(90.6 \%)$ & 3 & $27(84.4 \%)$ & 5 & $31(86.1 \%)$ & 5 \\
\hline$B$ & $\begin{array}{l}\text { DEA } 1 \\
\text { Strong + }\end{array}$ & $6(100 \%)$ & 0 & $4(80 \%)$ & 1 & $2(33.3 \%)$ & 4 \\
\hline$A B$ & $\begin{array}{l}\text { DEA } 1 \\
\text { Strong + }\end{array}$ & $6(100 \%)$ & 0 & $6(100 \%)$ & 0 & $5(83.3 \%)$ & 1 \\
\hline$A$ & $\begin{array}{l}\text { DEA } 1 \\
\text { Weak + }\end{array}$ & $17(77.3 \%)$ & 5 & $18(81.8 \%)$ & 4 & $18(75 \%)$ & 6 \\
\hline$B$ & $\begin{array}{l}\text { DEA } 1 \\
\text { Weak + }\end{array}$ & $2(100 \%)$ & 0 & $2(100 \%)$ & 0 & $2(100 \%)$ & 0 \\
\hline$A B$ & $\begin{array}{l}\text { DEA } 1 \\
\text { Weak + }\end{array}$ & $4(80 \%)$ & 1 & $5(100 \%)$ & 0 & $4(80 \%)$ & 1 \\
\hline$A$ & $\begin{array}{l}\text { DEA } 1 \\
\text { Negative }\end{array}$ & $20(80 \%)$ & 5 & $21(84 \%)$ & 4 & $18(72 \%)$ & 7 \\
\hline B & $\begin{array}{l}\text { DEA } 1 \\
\text { Negative }\end{array}$ & $4(100 \%)$ & 0 & $2(50 \%)$ & 2 & $1(25 \%)$ & 3 \\
\hline$A B$ & $\begin{array}{l}\text { DEA } 1 \\
\text { Negative }\end{array}$ & $3(100 \%)$ & 0 & $1(33.3 \%)$ & 2 & $1(33.3 \%)$ & 2 \\
\hline
\end{tabular}

15 